\title{
Un viaje por la movilidad cotidiana. E1 espacio entre el transporte y el individuo
}

\author{
A journey through daily mobility. The space between \\ transportation and the individual
}

Bruno Salerno ${ }^{1}$

\section{Resumen}

Este artículo construye un recorrido a lo largo de la producción actual de la movilidad cotidiana poniendo atención en el problema de la oferta y la demanda. En este sentido, se expone un avance progresivo desde enfoques más centrados en el transporte, hasta enfoques más orientados hacia el individuo. Este problema va de la mano de la tensión entre agregadoindividuo. Finalmente, la argumentación va a ser construida en clave de la relación entre la movilidad como una práctica social y las relaciones sociales y la producción del espacio en general. Las conclusiones versan, en gran medida, sobre las implicancias y supuestos de la adopción del individuo como objeto de estudio.

Palabras clave: Movilidad, espacio, transporte, individuo, oferta y demanda

1 Licenciado en Geografía por la Facultad de Filosofía y Letras, Universidad de Buenos Aires. Becario doctoral del Consejo Nacional de Investigaciones Científicas y Técnicas (CONICET). Doctorando en Geografía por la Facultad de Filosofía y Letras, Universidad de Buenos Aires. Filiación Institucional: Instituto de Geografía, Facultad de Filosofía y Letras, Universidad de Buenos Aires. País: Argentina. Correo electrónico: br.salerno@gmail.com 


\section{Abstract}

This article builds a route along the current status of daily mobility focusing in the problem of supply and demand. In that sense, we propose a progressive move from approaches focused on transport, to approaches concerned about the individual. This problem goes hand in hand with the tension between the aggregate and the individual. Finally, the argument will be built in the key of the relationship between mobility as a social practice and social relations, and the general production of space. The conclusions largely relate to the implications and assumptions of the adoption of the individual as the subject of study.

Keywords: Mobility, space, transport, individual, offer and demand. 


\section{Introducción}

El propósito de este artículo es presentar un análisis crítico de algunas líneas actuales de la producción académica sobre movilidad cotidiana. Se utiliza el modificador "cotidiana" para restringir el concepto a aquellas formas que se distinguen por su habitualidad, diferenciándose así de la movilidad por vacaciones, migraciones, o de otros conceptos, como es el caso de la movilidad social o de la movilidad residencial. De esta manera, si se considera que se pueden pensar los trabajos de movilidad agrupados en perspectivas macro (movilidad y cambio de la estructura social) y perspectivas micro (movilidad al interior de una formación social) (Widmer \& Schneider, 2006), en este trabajo nos iremos centrando progresivamente en este último grupo.

El eje que guía la exposición se estructura en torno al paso progresivo desde perspectivas que ponen énfasis en la oferta a perspectivas de la movilidad, que ponen énfasis en la demanda. Como se puede deducir de este planteo, en la bibliografía que se cita a lo largo de este trabajo, la movilidad aparece asociada al problema de la relación entre la oferta -aspecto más relacionado con el transporte- y la demanda -lado más asociado a las personas.

El problema de la vinculación entre la oferta y la demanda puede asociarse, en términos más abstractos, a la preocupación propia de la geografía de pensar el espacio como una instancia de las relaciones sociales. En este sentido, el estudio de la movilidad que propone el artículo se asocia al problema de pensar las relaciones complejas que se dan entre las prácticas sociales y la producción del espacio, y en términos más particulares, a la preocupación por la vinculación entre la movilidad de los habitantes de la ciudad y la producción del espacio urbano.

Al problema del peso de la oferta y la demanda va íntimamente ligado el del sujeto de la movilidad. Se puede pensar que este oscila, en los trabajos considerados, desde los agregados a los individuos. Mientras los trabajos sobre oferta tienden a pensar en agregados, los que se centran focalmente en la demanda van a hacerlo en términos de individuos. Esto, al mismo tiempo, va a ir de la mano del paso desde la consideración de la movilidad en términos de un fin exclusivo -el trabajo- a la movilidad asociada a diferentes fines.

¿Cuáles son las consecuencias de las tensiones entre oferta y demanda y entre agregados e individuos para el estudio de la movilidad y para pensar la vinculación entre la movilidad y las relaciones sociales en general y la producción del espacio en particular? Esta es una pregunta central sobre la cual pretendo proponer algunas reflexiones.

\section{La movilidad cotidiana y la oferta}

Se puede pensar que hay un conjunto de trabajos sobre movilidad -en términos amplios- que se refieren con mayor énfasis al problema de la oferta de movilidad que al de la demanda, aunque no se lo 
diga en esos términos. Es decir, al lado de las redes y al lado del transporte. Hay muchas maneras de trabajar este tema y hay muchos puntos grises en lo que refiere a la clasificación de estos trabajos. Se puede pensar, sin embargo, que hay por lo menos dos grupos de trabajos diferenciables. Por un lado, los trabajos enmarcados dentro de la geografía del transporte; por el otro, los trabajos que ponen énfasis en el problema de la accesibilidad. De hecho, hay múltiples intersecciones entre estos dos grupos. Sin embargo, parecería que el primer grupo tiene una entidad bien reconocible, mientras que el último, por su parte, es donde más difícil es establecer divisiones categóricas, ya que se encuentra fuertemente vinculado con otros estudios donde la demanda tiene un papel más importante.

\subsection{La geografía del transporte}

El de la geografía del transporte es uno de los campos donde más conscientemente se hacen preguntas sobre la relación entre las prácticas sociales y el espacio. En efecto, el problema de la causalidad entre la construcción de infraestructuras y sus "efectos territoriales" parece ser uno de los ejes que atraviesan las exposiciones (Keeling, 2007a-b).

Tradicionalmente la geografía del transporte se ha especializado, de la mano de las corrientes analíticas de la geografía, en los problemas de la localización y de las relaciones espaciales (Seguí \& Martínez, 2004), y los patrones y mag- nitudes de movimiento de los sistemas de transporte (Keeling, 2007a). Sin embargo, desde finales de la década del 80 , se ha desarrollado la preocupación por la vinculación del transporte con los procesos sociales. Mayoritariamente, estos trabajos se han centrado en la dimensión "macro" de la movilidad (Widmer \& Schneider, 2006), en términos de la vinculación del transporte con los cambios socioespaciales de las sociedades. Esto, independientemente de las problematizaciones de la vinculación entre estos dos temas, que ha dado lugar a planteos menos causales y más complejos (Miralles, 2002b; Seguí Pons \& Martínez, 2004) -en sintonía con lo que ha sucedido en la relación entre espacio y relaciones sociales en la geografía en general-. Ejemplos de los temas trabajados son la vinculación del transporte con la globalización y los cambios de las velocidades de los flujos de circulación, $\mathrm{y}$, a escala regional, las relaciones entre el transporte y el desarrollo económico de países o regiones (Keeling, 2007b). Otros estudios, ya a escala metropolitana, han analizado, como el caso de Blanco (2002) para las autopistas del norte del Área Metropolitana de Buenos Aires, la vinculación entre infraestructuras y patrones de urbanización.

Se ve en los trabajos recién citados que una variable de estudio importante para entender la relación entre transporte y el espacio es la escala. Esta perspectiva tiene la mira puesta, simultáneamente, en el fundamental problema de las políticas de transporte. 


\subsection{La accesibilidad}

Los trabajos sobre accesibilidad, por su lado, se distancian de las líneas más clásicas de la geografía del transporte, en el sentido de alejarse de las infraestructuras $y$ en incorporar elementos relacionales, sin dejar de centrarse en la oferta. Lévy (2000), de hecho, llama accesibilidad a la oferta de movilidad, y asocia ese concepto a la limitación -en términos de condición necesaria- de las posibilidades de la movilidad. En este sentido, este autor habla de movilidad como posibilidad.

La formulación del problema de la accesibilidad también se puede pensar desde el planteo de Lévy, en términos de que la oferta de movilidad se da mediante la oferta de transporte. Esto es lo que le permite a Lévy decir que la accesibilidad es la "dimensión material" de la movilidad, fórmula que tiene cierta correlación con la de Miralles (2002a), para quien la accesibilidad es "la dimensión espacial" de la movilidad.

En esta perspectiva aparecen los individuos de una forma más marcada que en la anterior. Sin embargo, esto no sucede más que en forma de agregados vinculados a los desplazamientos efectivos -en las redes-.

Simultáneamente, los trabajos sobre accesibilidad plantean una distinción entre dos problemas contrapuestos: la accesibilidad se puede pensar como atributo de los lugares o como atributo de las personas. En términos de la primera definición, hay lugares más o menos accesibles, en relación con la facilidad con la que se puede llegar a ellos. En segundo lugar, la accesibilidad se puede pensar como atributo de las personas, en términos de capacidad de acceso a los recursos. En esta segunda dimensión se mueven muchos de los trabajos de este campo y la preocupación de este artículo, si bien es cierto que las perspectivas son complementarias y necesarias en los estudios concretos. Kralich (1993, 2002, 2009a-b, 2011), por ejemplo, estudia la accesibilidad de la red de transporte metropolitano de Buenos Aires. Utiliza para esto algunos indicadores, como ejemplo las tarifas, el número de trasbordos y el tiempo de viaje. Entre estos elementos, el que ha sido privilegiado por la autora es el de las tarifas, que ella analiza en forma de "capital monetario".

En esta forma de pensar la noción, se torna menos claro el problema de la delimitación de campos de estudio, en términos de énfasis entre oferta y demanda. Lo que sí parece claro es que pensar la accesibilidad como un atributo de los individuos ya supone un distanciamiento de la oferta en términos más concretos y da lugar a su vinculación con los problemas de la demanda.

\section{De la oferta a la demanda}

Simultáneamente al desarrollo del campo de la geografía del transporte y de los trabajos sobre accesibilidad, existe una cantidad importante de producción que, desde diferentes perspectivas, aborda la relación entre la oferta y la demanda. Se van a distinguir dos grupos. 
En primer lugar, se pueden mencionar los trabajos de raíz cuantitativa, como es el caso de los relacionados con la ingeniería del transporte. Esta disciplina, que en un principio se ocupó principalmente de las infraestructuras y de los flujos, ha llegado a elevados niveles de modelización y abstracción incorporando el problema de la demanda. Entre los trabajos producidos desde estos enfoques, algunos de los más famosos son los asociados a los modelos macro de celdas de atracción que se trabajan con las matrices y encuestas de Origen y Destino (Hernández \& Witter, 2011). Sin embargo, como revelan Widmer y Schneider (2006), existe también una importante producción actual centrada en la escala micro, inclusive en relación con enfoques comportamentales, como pueden ser, por ejemplo, los trabajos que recurren a la teoría de la Rational Choice (por ejemplo, Cantillo \& De Dios, 2005). Mucha de esta producción es decisiva para la planificación de las redes de transporte

En segundo lugar, se puede hablar de la producción llevada a cabo desde enfoques más cualitativos de las ciencias sociales. Estos trabajos, desarrollados con fuerza en las dos últimas décadas, conforman un heterogéneo conjunto que muchos autores, como Miralles (2002a), van a describir como un nuevo paradigma. El nuevo paradigma, preocupado por las "movilidades diferenciales", se sostiene sobre una distinción fuerte entre la noción de movilidad y la de transporte. La primera de estas nociones es presentada, así, como un concepto más amplio que el de transporte, limitándose el rol de este último al de so- porte material de la movilidad, cuestión que va de la mano de un desdén relativo hacia los agregados, reforzando, como contrapartida, una particular preocupación por los viajes y sus motivaciones, si bien en un primer momento la motivación predilecta fue el trabajo.

Esta perspectiva tiene como objeto de sus críticas a los enfoques más tradicionales de la ingeniería del transporte. Un ejemplo puede verse en la siguiente cita:

Si desde la ingeniería tanto del tránsito como de transporte se consideraba iguales a todos los individuos, desde la óptica de la movilidad se considera la existencia de una multitud de sujetos diferenciados según la edad, el sexo, la clase social, el grupo étnico, la condición física o psíquica, etc. cada uno de los cuales tiene necesidades diferenciales de movilidad $\mathrm{y}$, consecuentemente, requiere de soluciones también diferenciadas. (Avellaneda, 2007, p. 118)

En el conjunto de la producción sobre movilidades diferenciales pueden reconocerse, sin embargo, muchos matices. Se puede advertir aquí también una tensión entre trabajos más centrados en la oferta -más preocupados por el tránsito, por ejemplo- (Sanz, 1997, 2005) y trabajos más enfocados en la demanda. Con relación a estos últimos, se puede decir que ha habido una tendencia a la focalización en las "poblaciones excluidas de la movilidad". Estos trabajos comenzaron a plantear más conscientemente el pro- 
blema de la relación entre la movilidad y las relaciones sociales, puntualmente en términos de la movilidad como un elemento central para la reproducción social, si bien la terminología varía. Al mismo tiempo se tendió a estudiar grupos sociales, en general subalternos (según nivel económico, género, o lugar de residencia por ejemplo). La producción desde este enfoque se desarrolla fuertemente en Francia (Díaz, Mignot \& Paulo, 2006; Díaz, Plat \& Pochet, 2007; Reobouha \& Pochet, 2009), España (Cebollada, 2006, 2009; Cebollada \& Avellaneda, 2008; Presseguer, 2005; Tineo I Marquet \& Camós I Cabeceran, 2005) y también Latinoamérica (Avellaneda, 2007; Avellaneda \& Lazo, 2011; Lazo, 2008).

\section{3. la movilidad como relación social: el centraje en el individuo}

En una búsqueda por abrir el horizonte de los estudios de movilidad, desde la década de 1990 se desarrolló, enmarcado en el problema de las poblaciones excluidas de la movilidad, un conjunto de trabajos, muchos de ellos en el ámbito francófono, orientados a profundizar en los aspectos asociados a la subjetividad y al individuo. Un trabajo importante y muy citado dentro de esta línea y de las líneas tributarias a esta, fue el de Lévy (2000), desde el cual se definió la movilidad como una relación social ligada al cambio de lugar, $\mathrm{y}$ en el cual nos detendremos.

Lévy piensa la movilidad como el conjunto de modalidades por las cuales los miembros de una sociedad tratan la posibilidad de ellos mismos o de otros de ocupar sucesivamente distintos lugares (Lévy, 2000). Es importante el juego de tres dimensiones que realiza este autor, y que permite ver cómo se estructura la idea de movilidad como relación social en él y en otros autores analizados en este trabajo. Para este autor, la movilidad se puede pensar en tres dimensiones. Una primera dimensión se relaciona con la oferta, y se trata de la movilidad como potencialidad. Esta acepción se conecta directamente con lo visto en la primera sección de este trabajo. Una segunda dimensión es la movilidad como competencia, y se relaciona con las competencias particulares del individuo para efectuar la movilidad. La tercera dimensión, que se analizará en la sección correspondiente, se refiere a la movilidad como capital. Lévy (2000) habla de una relación triangular entre lo deseado, lo posible y lo realizado.

Volviendo a la segunda dimensión de Lévy, se puede decir que la relación entre la movilidad ofrecida y la realizada requiere una competencia, dentro de la cual el autor da particular importancia a los medios materiales y financieros. El rol que juegan estos elementos se pone en práctica en el problema del acceso a los medios de transporte, pero también, en la constitución de una red de lugares frecuentados, ellos mismos situados en una buena posición en el espacio de las accesibilidades. Sin embargo, Lévy se cuida de alejar la idea de una relación lineal entre ingresos y movilidad: "la posesión de una competencia de movi- 
lidad es un componente, más que una consecuencia, de una posición social jerárquicamente inferior. En particular, es porque son poco móviles que las poblaciones desposeídas son desposeídas" (Lévy, 2000, p. 4).

En el problema de la competencia de movilidad, profundiza Le Breton (2002b), quien hace hincapié en el rol de los elementos individuales en las prácticas de movilidad. Para este autor, no obstante, no hay una competencia de movilidad, sino competencias en plural, en una fórmula que enriquece el enfoque. En este sentido, este autor muestra cómo hay cierto orden de elementos que están en la base de la movilidad y que son requeridos por ella. En términos generales, afirma que la movilidad está determinada por la forma en que los individuos se apropian del espacio. Entre los elementos destacados por el autor, resalta en primer lugar las competencias de los tipos físicos y culturales. Por ejemplo, en la ciudad hay una serie de códigos culturales que es necesario compartir para poder desplazarse. En segundo lugar, y con una centralidad manifiesta, destaca las representaciones del espacio: "tiene que haber una representación del territorio donde se va a efectuar el desplazamiento" (Le Breton, 2002b, p.3). Las representaciones van a condicionar las zonas que se puedan abarcar y los tipos de viaje que se lleven a cabo: "para las personas que nos interesan, la ciudad no es un territorio solidario, continuo, donde todo es potencialmente accesible. Por el contrario, es un territorio plagado de zonas a (sic) evitar, de zonas prohibidas, de contrastes, de territorios dolorosos. Estas fronteras limitan las movilidades" (Le Breton, 2002b, p. 4).

También son factores importantes la dinámica familiar y las convenciones culturales, este último aspecto central en lo que respecta al género -por ejemplo, las mujeres parecen enfrenar límites a la movilidad más marcados que los hombres- (Le Breton, 2002a).

En el ámbito latinoamericano, la producción de Andrea Gutiérrez (2009, 2010; Gutiérrez \& Minuto, 2007; Gutiérrez \& Rearte, 2006), sigue trabajando la línea marcada por estos autores. La autora en cuestión va a definir la movilidad como una performance en el territorio, en tanto una "práctica social de viaje". En su elaboración teórica, va a pasar a tener centralidad como objeto de estudio el viaje. En total sintonía con los trabajos francófonos, esta definición permite oscilar entre la dimensión material de los desplazamientos efectivamente realizados hacia la capacidad efectiva de su realización. Los viajes están motivados por la necesidad y el deseo; y la movilidad, entonces, se asocia a la capacidad de satisfacerlos.

La consideración de las cuestiones del deseo permite abrir el juego de las motivaciones de los viajes. Si en los primeros estudios que ponían en relación oferta y demanda predominaba el trabajo como fin del viaje, en estos se abre el panorama no solo a los recursos que ofrece la ciudad y que son requeridos para la reproducción de las personas, sino también a 
los deseos, multiplicándose el número de motivaciones. A esto se alude, por otro lado, con el uso en plural que hace Lévy de la noción, se trata de movilidades.

Existiría de esta manera una serie de mediaciones entre la movilidad y el viaje efectivamente realizado, retomando las ideas de Le Breton (2002b): desde la movilidad concebida por el sujeto, pasando por la que considera que se puede realizar hasta la elección y realización de una. Esto lleva a que se considere que existe una dimensión de viajes no realizados, pero también de viajes pensados $\mathrm{o}$ viajes posibles.

Los trabajos sobre movilidad como capital van a complejizar estos planteos, que si bien presentan cierto grado de heterogeneidad apuntan a que hay ciertos elementos en el orden del individuo que se juegan en el problema de la movilidad. En el contexto de este planteo, se puede proponer un problema que nos va acompañar en la próxima sección: ¿Cómo se puede analizar la movilidad en tanto una relación social, como pretenden Lévy, Le Breton o Gutiérrez en los trabajos citados, pensando al individuo como unidad de análisis?

\section{La movilidad como capital}

La idea de pensar la movilidad como capital se vincula a los desarrollos sobre la noción de capital social, fundamentalmente, con base en los trabajos de Bourdieu (1986). Sin embargo, si se analizan con detenimiento los desarrollos de este autor, y de los trabajos sobre capital de movilidad, se puede ver que lo que se va tomar en este último campo es la idea del capital como un conjunto de recursos presentes y potenciales necesarios para llevar a cabo los viajes (tangibles y menos tangibles; dinero, competencias culturales, etc.), dejándose de lado la cuestión de la pertenencia a un grupo y la existencia de relaciones de intercambio material y simbólico al interior de ese grupo. En la idea de Bourdieu ${ }^{2}$, los recursos se encuentran ligados a estas relaciones.

Centrándonos ahora en el campo de la movilidad, retomamos la idea de la tercera dimensión de movilidad analizada por Lévy (2000). Para este autor, "el conjunto constituido por la posibilidad, por la competencia y por los arbitrajes que la segunda permite sobre la primera puede ser leído como un capital social, un bien que permite al individuo desdoblar mejor su estrategia al interior de la sociedad" (Lévy, 2000, p. 5).

Si para Lévy la posibilidad se refiere a la oferta, y las competencias se juegan en el plano de las capacidades del individuo, se entiende cómo la idea de capital se presenta como un conjunto abarcativo que incluye cuestiones de la oferta y la demanda. Esto se puede ver en el desarrollo teórico de Arce y Soldano (2010), en un trabajo que analiza un caso de la periferia del Área Metropolitana de Buenos Aires.

2 Ramírez Plascencia (2005) muestra las diferencias de la noción de capital social en los trabajos de Bourdieu con relación a otros autores como Coleman y Putman. Por cuestiones de espacio y de propósito del artículo no se puede desarrollar este tema aquí. 
Estas autoras introducen la noción de capital espacial, en términos del espacio como un bien social del cual los individuos hacen uso, gracias a la experiencia de la ciudad. En este sentido, este capital incluiría dos subtipos: capital de posición y capital de situación. El primero se vincularía con la dimensión de posibilidad de capital de Lévy, mientras que el segundo se asociaría con la dimensión de competencia. Es decir, uno se asociaría a la oferta y otro a la demanda. El capital de movilidad propiamente dicho, que en Lévy pone en relación las dos otras dimensiones, en el trabajo de las autoras aparece, en una fórmula no del todo clara, como un tipo de capital de situación.

Los conceptos así desarrollados les permiten a las autoras, en el análisis del caso, por un lado analizar la oferta de transporte y de infraestructura con el origen de coordenadas en la residencia, ya que se trata del análisis de la movilidad de una población particular. Así, se analizan las características de la red asfáltica, y la provisión de redes, en términos de accesibilidad. Por otro lado, yendo al capital de situación, las autores terminan analizando elementos que se pueden pensar en términos de recursos individuales: posesión de automóvil, dinero, etc.; elementos que terminan vinculando con el primer grupo en forma de tiempo y costo de viaje.

La idea de movilidad como capital, al igual que la segunda dimensión de Lévy, tiene el mérito de relacionar, más que de separar, elementos de diferentes enfoques sobre movilidad, en la medida en que permite unir bajo una misma matriz diferentes áreas de análisis. Sin embargo, volviendo a la definición de Bourdieu, se puede pensar que algo se pierde en el traslado de la noción de capital social al campo de movilidad. Vemos con Ramírez Plascencia que "el volumen de capital social al alcance de un individuo depende no sólo de la red de conexiones que posea, sino 'del volumen de capital (económico, cultural o simbólico) poseído por aquellos con quienes está relacionado"” $(2005$, p. 5$)$.

El énfasis en el individuo que marca la entrada en la perspectiva de la movilidad como relación social, de la cual la del capital de movilidad es tributaria, tiene el mérito de recuperar una dimensión de problemas no trabajada previamente (los asociados a la demanda en términos de los individuos). Esto, sin embargo, le genera limitaciones, en este caso, la imposibilidad de incorporar teóricamente toda la complejidad de la noción original de capital social.

\subsection{La motilidad}

Otro conjunto de autores ha dado un paso más en el desarrollo teórico sobre la idea de capital de movilidad, al punto de proponer otro concepto, el de motilidad, para referirse a este tema. Un trabajo importante en esta dirección es el de Kaufmann, Bergman y Joye (2004).

Como señalan Widmer y Schneider (2006, p. 28), "estrictamente hablando, la motilidad no trata de explicar la movilidad. Más bien, es un concepto que 
pretende reemplazar la movilidad como objeto de interés de las ciencias sociales". Se trata de una construcción teórica que pretende englobar a la movilidad, que aparece de esta manera como una manifestación de la motilidad.

La motilidad es la capacidad de una persona de ser móvil. En este sentido se vincula fuertemente con lo expuesto sobre capital de movilidad, en relación con el conjunto de elementos que hacen posible la movilidad. Sin embargo se diferencian de esta concepción al reenfocar el problema de estudio: se busca el distanciamiento del desplazamiento o del viaje en sí mismo y se focaliza aún más en el individuo, vinculándose al problema de la potencialidad de la movilidad (Kaufmann, Bergman \& Joye, 2004, p. 5). En este sentido, el de motilidad se propone como un término que llega para desambiguar y precisar los problemas que engloba el término movilidad. Así, la motilidad, pese a incluir consideraciones sobre la oferta, es en gran medida un atributo del individuo.

Un elemento interesante de Kaufmann, Bergman y Joye, es que parecería verse una voluntad de producir una teoría que intenta repensar la vinculación entre movilidad y relaciones sociales, con el fin de situar al individuo en una trama social. En este sentido los autores proponen su teoría como una forma de intentar vincular el problema de la movilidad espacial con el de la movilidad social. Siguiendo esta línea, pretenden que la movilidad sea concebida como un tipo de capital más, apropiable, junto con otros tipos de capitales sociales, culturales, o de otro tipo, pudiéndose intercambiar y relacionar con estos. La forma concreta en que esto puede pensarse no es sin embargo clara.

Para pensar los factores que se encuentran detrás de la motilidad, finalmente, Kaufmann, Bergman y Joye van a proponer tres categorías: el acceso, las competencias y la apropiación, inspirándose en las tres de dimensiones de Lévy (2000).

Se puede dar todavía una vuelta más al asunto. Jouffe (2011), en una intrincada teorización, se sostiene críticamente sobre el trabajo de Kaufmann, Bergman y Joyce para repensar la idea de capital de movilidad. En este sentido, el autor va a afirmar que la movilidad no es un capital sino un "para-capital" al servicio de otros capitales. Por ejemplo, dice el autor, el para-capital de movilidad es una herramienta de lucha, y el hábitat es el objetivo de esta lucha.

Más allá de las perspectivas concretas, es interesante ver la profundidad de las preguntas que se han podido sostener sobre la idea de capital y cómo se ha producido una profundización del centraje en el individuo, o dicho de otra manera, un alejamiento del problema de la oferta. Simultáneamente, se puede ver, como si se estuviera intentando responder la pregunta planteada oportunamente, que se ha desarrollado una creciente preocupación por vincular la movilidad con preguntas del ámbito de las relaciones sociales. 


\section{Movilidad, espacio y territorio}

Las corrientes críticas en geografía, desde su origen en la década del 70 , han enfatizado la compleja relación entre espacio y relaciones sociales. Los trabajos ahora clásicos de Castells (1974), Harvey (1985) y Lefebvre (2009), originalmente publicados en 1972, 1973 y 1974 respectivamente, fueron los primeros en proponer un análisis riguroso orientado a desfetichizar la primera de estas nociones. Se introdujo el concepto de producción del espacio, que daba cuenta de su carácter social, frente a corrientes previas donde aparecía naturalizado. En una segunda instancia se complejizó esta definición. Blanco (2007, p. 38) sintetiza esta reformulación: "el espacio participa como condicionante de los procesos sociales al mismo tiempo que como su producto, en una secuencia de opuestos como productor-producido, subordinante-subordinado, presupuestoconcreción".

Esta compleja relación, a la que también aludió Bourdieu (2010), abarca el par movilidad-espacio, que en muchas ocasiones, sobre todo cuando se reduce el problema al transporte, se ha considerado más lineal. En este sentido, retomando las consideraciones hechas en relación con la geografía de transporte, no se puede reducir la relación entre estos dos elementos ni en términos de estructuras espaciales como determinantes de las formas de movilidad, ni de movilidad como estructurante del espacio. En el marco de su consideración como una instancia de las relaciones sociales, cuya relación con ellas escapa a una causalidad lineal, el espacio debe ser considerado en su relación con la movilidad como parte de un proceso dialéctico de adaptación recíproca (Miralles, 2002b).

Una relación interesante entre movilidad y espacio existe en los que se centran en el problema de la elección residencial y en el de derecho a la ciudad, tema que por cuestiones de espacio no se desarrollará aquí. Si bien no hay muchos trabajos al respecto (por ejemplo, Contreras Gatica, 2011), este enfoque permite buscar formas más directas de relacionar la producción del espacio con la movilidad. Algunas consideraciones referidas a este tema se han realizado desde otros ámbitos, como es el caso de los estudios de mercados inmobiliarios informales, donde el problema se ha pensado en términos del peso de los factores exógenos y endógenos en el desarrollo de los mercados inmobiliarios de las urbanizaciones informales. De esta manera, factores exógenos como la cercanía a recursos específicos o equipamientos colectivos se vincula directamente con los precios inmobiliarios y la elección residencial (ver, por ejemplo, Abramo, 2003).

Otra de las formas en que se pueden vincular la movilidad y el espacio es por medio de la producción de territorios, noción que permite jugar con la materialidad e incluir cuestiones relacionadas con lo cultural, lo identitario y lo simbólico, entre otras. Cabe señalar que, como se sabe, el territorio de por sí es una categoría con un importante trasfondo teórico. Aquí se va a entender por te- 
rritorio el problema de la apropiación y delimitación de un ámbito sobre el que se posee control. La vinculación de esta perspectiva con el poder es fundamental.

\subsection{Espacio vivido y territorios cotidianos}

La puesta en relación de la movilidad como una relación social con el problema de los territorios es frecuente en muchos trabajos. La separación en esta otra sección se puede pensar más bien de forma analítica y parece justificada por la vastedad del campo de trabajo que abre.

La centralidad que da Le Breton a la movilidad en el contexto de las relaciones sociales, es lo que le permite vincularla con la producción de territorios. En efecto, en la medida en que la movilidad es "una dimensión transversal de la vida cotidiana, una matriz de todas las experiencias sociales" (Le Breton, 2006, p. 26), se puede pensar el problema de "cómo los individuos y los grupos sociales construyen sus identidades territoriales" (Le Breton, 2006, p. 24). Dicho en otros términos, los territorios de la cotidianeidad se refieren a los territorios producto de la apropiación cotidiana, a los territorios del espacio vivido -la "ciudad vivida", según el autor-, a los "territorios practicados".

Estos dos últimos conceptos tienen mucho que ver con las nociones de espacio de vida y espacio vivido, como las trabaja Guy Di Meo (2000). Mientras que el espacio de vida se asocia a la materialidad cotidiana, la idea de espacio vivido se refiere a la representación de la realidad espacial. Esto da pistas para pensar la vinculación entre territorio e identidad, problema que este mismo último autor piensa poniendo énfasis en la idea de apropiación. El grupo social que se apropia del espacio, construyendo el territorio, se da a sí mismo una representación particular. Por lo que el territorio termina implicando: en primer lugar, la inserción de los individuos en los grupos, lo que se vincula a la construcción de la pertenencia y la identidad colectiva; en segundo lugar, el control del espacio apropiado garantiza la especificidad, la permanencia y la reproducción del grupo; y en tercer lugar, construir un territorio implica organizar un campo simbólico que funda o reafirma la identidad del grupo en cuestión.

Otro aporte interesante presentado por Le Breton (2006) en relación con este tema, es el de los objetos intermediarios, elementos (por ejemplo, estaciones, escaleras mecánicas, teléfonos públicos) que son utilizados por las personas como parte de un proceso de articulación entre movilidad y territorios.

Gutiérrez (2010), finalmente, también se adentra en este tema, intentando combinar el problema de la movilidad como relación de fines y capacidades con el problema de los sentidos que da el sujeto al movimiento y al espacio en que se da el movimiento. La idea central de la autora, en lo que propone como una geografía del acceso, es que el acceso puede unir lugares conforme a un sentido o significado. Este sentido o significado se conectaría con el núcleo de sus desarrollos: el de 
concretar o satisfacer el fin que motiva el viaje. La morfología de este territorio del sentido y de la experiencia sería el objeto de estudio del enfoque que Gutiérrez propone.

\section{Conclusiones}

El recorrido que se siguió a lo largo de este artículo fue uno entre muchos otros posibles. La mirada que guió el trabajo, dejó de lado muchos problemas y catalogó y definió grupos de trabajos heterogéneos, muchas veces ignorando grises e imponiendo divisiones analíticas.

Entre los temas que no se trataron son interesantes los relacionados con el problema de captar nuevos fenómenos de la sociedad, como son los relacionados con las metáforas de la fluidez o la velocidad, o con nuevas formas de pensar el espacio urbano (Ascher, 2005, 2006). Estos fenómenos se captan sobre todo desde perspectivas macro, a las cuales, sin embargo, algunos autores que trabajan en perspectivas micro aluden (por ejemplo Lévy, 2000).

Al mismo tiempo, otras nociones de movilidad aparecen en la bibliografía: movilidad sostenible, movilidad obligada, y un número importante de conceptos que requieren ser pensados y problematizados.

En relación con el recorrido efectivamente realizado, la tesis que se sostiene a partir de la exposición es que la adopción del individuo como objeto de estudio fue una forma particular en la que se produjo la problematización de la demanda. ¿Había otra manera de pensar la demanda, por fuera de los trabajos más cercanos al tránsito (en sentido amplio)? Otra pregunta clave en relación a este tema sería: ¿Cuáles son las implicancias teóricas y los supuestos detrás de la consideración del individuo como objeto de estudio?

En primer lugar, la objeción planteada en su momento parece válida: ¿cómo se concilia el centraje en el individuo con pensar la movilidad como una relación social? Esto es crucial si se considera que el espacio no lo producen individuos aislados sino relaciones sociales. ¿En qué punto el centraje en el individuo nos desvía de aspectos centrales sobre la relación entre espacio y movilidad que sí son analizados desde la perspectiva de la geografía del transporte?

Como se comentó, la noción de capital de movilidad y de motilidad intenta pensar algunos de estos temas. Sin embargo, la solución no deja de presentarse, en estos trabajos, del lado del individuo. ¿Cómo se define este individuo de la movilidad? Quizás la solución sí esté de este lado, pero pensando en qué punto son recuperables los aspectos más relacionales de los desarrollos sobre capital social.

En este sentido, es interesante notar que se puede ver una convergencia entre el planteo amplio del capital social y la idea de soporte al estilo de Castel (por ejemplo, 2009). En esta línea de reflexión, 
que requiere un trabajo completo de reflexión, el estudio del individuo se asociaría al problema de la individuación.

Finalmente, a las preguntas ya planteadas sobre la relación con el espacio, parece muy interesante agregar las reflexiones sobre el tema de los territorios cotidianos y de los aspectos subjetivos. Sin embargo, más allá de los problemas que se relacionan con el centraje en el individuo, parece todavía un desafío pensar las formas de concretización de estas ideas de tan elevado nivel de abstracción. 


\section{Literatura citada}

Abramo, P. (2003). La teoría económica de la favela: cuatro notas sobre la localización residencial de los pobres y el mercado inmobiliario informal. Ciudad y Territorio: Estudios territoriales, número único, 136-137, 273-294.

Arce, C. P. \& Soldano, D. (2010). Capital espacial y movilidad cotidiana en la Región Metropolitana de Buenos Aires. Una propuesta analítica y empírica. En A. Rofman (comp.), Sociedad y territorio en el conurbano bonaerense. Un estudio de las condiciones socioeconómicas y sociopolíticas de cuatro partidos: San Miguel, José C. Paz, Moreno y Morón. Los Polvorines: Universidad Nacional de General Sarmiento.

Ascher, F. (2005). Los nuevos principios del urbanismo. El fin de las ciudades no está a la orden del día. Madrid: Alianza Editorial.

Ascher, F. (2006). El movimiento en el corazón de la modernidad. La urbanidad latente en los espacios del desplazamiento. Café de las Ciudades, 44. Recuperado de http:// www.cafedelasciudades.com.ar/tendencias_44.htm.

Avellaneda, P. (2007). Movilidad, pobreza y exclusión social. Un estudio de caso en la ciudad de Lima. Tesis doctoral no publicada. Departamento de Geografía, Universitat Autónoma de Barcelona.

Avellaneda, P. \& Lazo, A. (2011). Aproximación a la movilidad cotidiana en la periferia pobre de dos ciudades latinoamericanas. Los casos de Lima y Santiago de Chile. Revista Transporte y Territorio, 4, 47-58. Recuperado de www.rtt.filo.uba.ar/ RTT00404047.pdf

Blanco, J. (2002). La red de autopistas de Buenos Aires y la reorganización del espacio metropolitano en la década de 1990. Estudio de caso: el Acceso Norte ramal Pilar. Buenos Aires: Mimeo.

Blanco, J. (2007). Espacio y territorio: elementos teórico-conceptuales implicados en el análisis geográfico. En M. V. Fernández \& R. Gurevich (coord.), Geografía. Nuevos temas, nuevas preguntas. Un temario para su enseñanza. Buenos Aires: Biblos.

Bourdieu, P. (1986). The forms of capital. En J. Richardson (Ed.), Handbook of Theory and Research for the Sociology of Education. New York: Greenwood. Recuperado de http://www.marxists.org/reference/subject/philosophy/works/fr/ bourdieu-formscapital.htm 
Bourdieu, P. (2010). Efectos de lugar. En P. Bourdieu (dir.), La miseria del mundo. Buenos Aires: Fondo de Cultura Económica.

Cantillo, V. \& De Dios, J. (2005). A semi-compensatory discrete choice model with explicit attribute thresholds of perception. Transportation Research, 39, (7), 641-657.

Castel, R. (2009). La metamorfosis de la cuestión social. Una crónica del salariado. Buenos Aires: Paidós.

Castells, M. (1974). La cuestión urbana. Madrid: Siglo XXI.

Cebollada, A. (2006). Aproximación a los procesos de exclusión social a partir de la relación entre el territorio y la movilidad cotidiana. Documents d'Anàlisi Geogràfica, 48.

Cebollada, A. (2009). Mobility and labour market exclusion in the Barcelona Metropolitan Region. Journal of Transport Geography, 17, 226-233.

Cebollada, A. \& Avellaneda, P. (2008). Equidad social en movilidad: reflexiones en torno a los casos de Barcelona y Lima. Scripta Nova. Revista electrónica de Geografía y Ciencias Sociales, XII, 270 (47). Recuperado de http://www.ub.edu/geocrit/ sn/sn-270/sn-270-47.htm

Contreras, Y. D. (2011). Elecciones y anclajes residenciales de los nuevos habitantes del centro de Santiago: la experiencia del barrio Bellas Artes. Revista Transporte y Territorio, 4, 47-58. Recuperado de www.rtt.filo.uba.ar/RTT00404047.pdf

Di Meo, G. (2000). Que voulons-nous dire quand nous parlons d'espace. En J. Lévy \& M. Lussault. Logiques de l'espace, esprit des lieux. Géographies à Cerisy. París: Belin.

Díaz, L., Mignot D. \& Paulo C. (2006, 25-27 septiembre). Movilidad y desigualdades sociales y territoriales. I Congreso Internacional Los Ciudadanos y la Gestión de la Movilidad, Madrid, (paper).

Díaz, L., Plat, D. \& Pochet, P. (2007). Caminar y nada más. Movilidad diaria y pobreza en las ciudades subsaharianas. Scripta Nova. Revista electrónica de Geografía y Ciencias Sociales, XII, 261. Recuperado de http://www.ub.es/geocrit/sn/ sn-261.htm

Gutiérrez A. \& Rearte, J. (2006). Segregación y accesibilidad a servicios públicos de transporte en la Ciudad de Buenos Aires. En A. Brasileiro \& P. Marques da Silva (eds.). Panorama Nacional da Pesquisa em Transportes 2006. Rio de Janeiro: ANPET.

Gutiérrez, A. \& Minuto, D. (2007, 18-23 noviembre). Una aproximación metodológica 
al estudio de lugares con movilidad vulnerable. XIV Congreso Latinoamericano de Transporte Público y Urbano, Río de Janeiro, (paper).

Gutiérrez, A. (2009, 31-3 marzo-abril). Movilidad o inmovilidad: ¿Qué es la movilidad? Aprendiendo a delimitar los deseos. XV Congreso Latinoamericano de Transporte Público y Urbano, Buenos Aires.

Gutiérrez, A. (2010). Movilidad, transporte y acceso: una renovación aplicada al ordenamiento territorial. Scripta Nova. Revista electrónica de Geografía y Ciencias Sociales, XIV, 331 (86). Recuperado de http://www.ub.es/geocrit/sn/sn-331/sn-331-86.htm

Harvey, D. (1985). Urbanismo y desigualdad social. Madrid: Siglo XXI.

Hernández, D. \& Witter, R. (2011). Entre la ingeniería y la antropología: hacia un sistema de indicadores integrado sobre transporte público y movilidad. Revista Transporte y Territorio, 4, 29-46. Recuperado de www.rtt.filo.uba.ar/RTT00403029.pdf

Jouffe, Y. (2011). Las clases socio-territoriales entre movilidad metropolitana y repliegue barrial. ¿Tienen los pobladores pobres una movilidad urbana de clase? Revista Transporte y Territorio, 4, 84-117. Recuperado de www.rtt.filo.uba.ar/RTT00406084.pdf

Kaufmann, V., Bergman, M. M., \& Joye, D. (2004). Motility: Mobility as Capital. International Journal of Urban and Regional Research, 28, 4.

Keeling, D. J. (2007a). Transportation Geography-new directions on well-worn trails. Progress in Human Geography, 31 (2), 217-225.

Keeling, D. J. (2007b). Transportation Geography-new regional mobilities. Progress in Human Geography, OnlineFirst. Recuperado de http://phg.sagepub.com/content/ear ly/2007/12/18/0309132507084400.extract.

Kralich, S. (1993). Accesibilidad hogar-trabajo en el Gran Buenos Aires. Un estudio de caso en el partido de La Matanza. Revista Territorio, 6.

Kralich, S. (2002). La ecuación 'más privado menos estatal': quienes ganan y quienes pierden. El caso del servicio de transporte público en el Gran Buenos Aires. Eure, 28, 84. Recuperado de http://www.scielo.cl/scielo.php ?script $=$ sci_arttext\&pid=S0250$71612002008400005 \& \operatorname{lng}=\mathrm{es} \& \mathrm{nrm}=$ iso.

Kralich, S. (2009a). La accesibilidad en la ciudad dual. El caso de la Región Metropolitana de Buenos Aires. Revista Urbanística PVS. Periódico internacional de cultura y planificación urbanística en países en vías de desarrollo, numero speciale 20082009. [CD-ROM].

Kralich, S. (2009b, 4-6 de noviembre). Procesos de urbanización y movilidad cotidiana en la 
ciudad posfordista. El caso de la Región Metropolitana de Buenos Aires. X Jornadas Argentinas de Estudios de Población. San Fernando del Valle de Catamarca, Argentina.

Kralich, S. (2011, septiembre). Vaivenes y pormenores de la accesibilidad en la Región Metropolitana de Buenos Aires (RMBA). Revista La Gran Ciudad. En prensa.

Lazo, A. (2008). Transporte, movilidad y exclusión. El caso de Transantiago en Chile. Actas del X Coloquio Internacional de Geocrítica. Diez años de cambios en el mundo, en la geografía y en las ciencias sociales. Barcelona, 25-30 mayo. Recuperado de http://www.ub.es/geocrit/-xcol/64.htm

Le Breton, H. (2002a) La mobilité quotidienne dans la vie precaire. Synthèse. Note de recherche pour L'Institut pour la ville en mouvement - PSA Peugeot- Citroën et Abeille Aide et Entraide. Recuperado de http://www.ville-en-mouvement.com/articles.htm

Le Breton, H. (2002b). Les raisons de l'assignation territoriale. Quelques éléments d'appréhension des comportements de mobilité de personnes disqualifiées. Recuperado de http://www.ville-en-mouvement.com/articles.htm

Le Breton, H. (2006). Homo mobilis. En M. Bonnet \& P. Aubertel. La ville aux limites de la mobilité. Paris: Presses Universitaires de France.

Lefebvre, H. (2009a). La production de l'espace. París: Economica-Anthropos.

Lévy, J. (2000). Les Nouveaux Espaces de la Mobilité. En M. Bonnet \& D. Desjeux. Les Territoires de la Mobilité. Paris: Presses Universitaires de France.

Miralles, C. (2002a). La movilidad cotidiana. En Ciudad y territorio. Barcelona: Ariel.

Miralles, C. (2002b). Transporte y territorio urbano. Del paradigma de la causalidad al de la dialéctica. Documents d'Anàlisi Geogràfica, 41.

Presseguer, M. (2005). La movilidad laboral en Osona. En Diputació de Barcelona, Mobilitat obligada. SAM. Recuperado de www1.diba.cat

Ramírez, J. (2005). Tres visiones sobre Capital Social: Bourdieu, Coleman y Putnam. Acta Republicana. Política y Sociedad, 4, 4.

Rebouha, F. \& Pochet, P. (2009). Mobilité et accès à la ville pour le travail des populations pauvres du périurbain d' Oran. Cahiers de Méditerranée, 78. Recuperado de http:// cdlm.revues.org/index4706.html

Sanz, A. (1997). Movilidad y accesibilidad: un escollo para la sostenibilidad urbana. Cons- 
trucción de la Ciudad Sostenible, Documentos. Recuperado de http://habitat. aq.upm.es/cs/p3/a013.html

Sanz, A. (2005). El viaje de las palabras. En Diputació de Barcelona, Mobilitat obligada. SAM. Recuperado de www1.diba.cat

Seguí, J. \& Martínez, M. R. (2004). Geografía de los transportes. Palma de Mallorca: Universitat de les Illes Balears.

Tineo I Marquet, J. A. \& Camós I Cabeceram, J. M. (2005). La accesibilidad a zonas de baja densidad. En Diputació de Barcelona, Mobilitat obligada. SAM. Recuperado de www1.diba.cat

Widmer, E. \& Schneider, N. F. (eds.) (2006). State-of-the-Art of Mobility Research. A Literature Analysis for Eight Countries. Job Mobilities Working Paper, 2006-1. EU: Job Mob and Fam Lives. Recuperado de www.jobmob-and-famlives.eu

Recepción: 4 de octubre de 2011

Evaluación: 9 de diciembre de 2011

Aprobación. 8 de febrero de 2012 